\title{
Reference Values for Fitness Level and Gross Motor Skills of 4-6-Year-Old Chilean Children
}

\author{
Andrés Godoy-Cumillaf ${ }^{1}$ (D), José Bruneau-Chávez ${ }^{1}$, Paola Fuentes-Merino ${ }^{1}$, \\ Jaime Vásquez-Gómez ${ }^{2} \mathbb{D}$, Mairena Sánchez-López ${ }^{3,4}{ }^{\mathbb{D}}$, Celia Alvárez-Bueno ${ }^{3, * \mathbb{D}}$ \\ and Iván Cavero-Redondo ${ }^{3}$ iD \\ 1 Grupo de Investigación en Educación Física, Salud y Calidad de Vida, Facultad de Educación, \\ Universidad Autónoma de Chile, 4810101 Temuco, Chile; andres.godoy@uautonoma.cl (A.G.-C.); \\ jose.bruneau@uautonoma.cl (J.B.-C.); paola.fuentes@uautonoma.cl (P.F.-M.) \\ 2 Vicerrectoría de Investigación y Postgrado, Centro de Investigación en Estudios Avanzados del \\ Maule (CIEAM), Universidad Católica del Maule, 3460000 Talca, Chile; jvasquez@ucm.cl \\ 3 Health and Social Research Center, Universidad de Castilla-La Mancha, 16071 Cuenca, Spain; \\ mairena.sanchez@uclm.es (M.S.-L.); ivan.cavero@uclm.es (I.C.-R.) \\ 4 Faculty of Education, Universidad de Castilla-La Mancha, 13071 Ciudad Real, Spain \\ * Correspondence: Celia.alvarezbueno@uclm.es
}

Received: 17 December 2019; Accepted: 25 January 2020; Published: 28 January 2020

check for updates

\begin{abstract}
In childhood, fitness level is considered an important indicator of health, while gross motor skills are the basis of future motor competence. So far, no reference values have been found for the Chilean population. Therefore, this study aims to provide fitness level and gross motor skill reference values by gender and age of Chilean children aged 4-6 years. A cross-sectional analysis was conducted that included 728 children between 4 and 6 years old from the La Araucanía region of Chile. To assess the fitness level, the 20-m shuttle run test, standing long jump, handgrip dynamometry, $4 \times 10 \mathrm{~m}$ shuttle run, and Sit and Reach tests were used. Gross motor skills were assessed by five tests including aiming and catching and balance motor tasks. For fitness level, boys have better values in the long jump and dynamometry test, while girls have better values in flexibility. For estimated maximum volume of oxygen, at 5 years old there are significant differences in favour of boys, while at 6 years old in favour of girls. No statistically significant differences in speed/agility by gender were found. For gross motor skills, boys obtain higher values for catching and aiming tests, and girls for balance. The reference values for fitness level and gross motor skills shown in this study could aid physical education and health professionals in identifying children with low reference values, as well as in establishing objectives that will help to improve their health.
\end{abstract}

Keywords: motor competence; strength; speed/agility; cardiorespiratory fitness; flexibility; balance; aiming; catching

\section{Introduction}

High fitness level is considered an important indicator of health in childhood [1,2], while low fitness level is associated with an increased risk of acquiring cardiovascular diseases in adulthood [3-5]. Moreover, cardiorespiratory fitness is a basic component of a healthy lifestyle [6,7], its optimum levels being associated with improved cardiovascular, skeletal, and mental health [1,2]. It should be highlighted that both fitness level and cardiorespiratory fitness depend on genetic factors [8], including anatomical and physiological, and environmental factors [9], such as behaviour and lifestyle aspects. For muscular strength from childhood to adolescence, the increased levels are associated with a decrease in total adiposity [1] and lower metabolic risk [10-12]. Likewise, speed/agility improvements 
are related to higher bone mineral density and accumulation of bone mass in later stages of life [4,5]. Also, flexibility plays an important role in the range and coordination of movements, this ability being associated with good results in terms of fitness level [13]. Considering everything mentioned above, previous studies [14] have suggested that it is necessary to include tests to assess the fitness level of children in the school settings, taking even more into account that Chile lacks records on indicators of fitness level [15], presenting a considerable gap in the literature for children 12 years old or younger [16].

Motor competence, defined as the degree of performance in a range of motor tasks that are rated on good control and movement coordination [17], is important because of its observed implications in the physical, mental, and social development of children and adolescents [18,19]. Motor competence is composed of fine and gross motor skills, with the former being important in the academic and social fields [18], and the latter developing the basis of future motor competence. Therefore [20], if children lack mastery in these skills, it is probable that they will have limited opportunities to successfully participate in the different physical activities throughout life [21], and as a result, delays or deficiencies in gross motor skills may affect children and adults' fitness levels [22].

In considering early prevention of risk factors for noncommunicable diseases (obesity, diabetes, cardiovascular disease, and others), assessment of fitness levels and gross motor skills is necessary for decision-making and intervention aimed at promoting healthy behaviour. These assessments require the existence of updated reference values that allow the categorization of individuals and groups according to fitness levels and gross motor skills. Additionally, these values might be established by sex as previous evidence has demonstrated significant differences in fitness levels and gross motor skills between boys and girls at early ages [23-26].

For 4-6-year-old children, there are international established reference values, for both fitness level $[14,27,28]$ and gross motor skills [22,29]. However, until now there is no clear cut-off for the Latin American population, specifically Chile, which makes it necessary to have their own values due to sociodemographic factors, the environment, and adequate nutrition of this population, which lead them to have different characteristics. Therefore, the present study aims to describe fitness levels and gross motor skills by gender and age, and to provide the first reference values in children aged 4-6 years, from La Araucanía Region, Chile.

\section{Methods}

This is a cross-sectional analysis of data from baseline measurements of a randomized controlled trial (RCT) registered in ClinicalTrial.gov (NCT04194580), aimed at assessing the effectiveness of a physical activity intervention in preventing and treating obesity and excess weight in children aged 4-6 years. The RCT study included 836 schoolchildren from the city of Pitrufquén, La Araucanía region, Chile. For the current study, data from 728 (87\%) children who had valid fitness level and gross motor skill measurements were used (Figure 1). The exclusion criteria were: (i) children not having legal guardian consent or not having the child's consent to carry out evaluations; (ii) children having some type of physical and/or mental disorder; and (iii) children suffering a chronic illness that could prevent participation in the physical activity intervention. The study was approved by the Scientific Ethical Committee of the Universidad Autónoma de Chile ( $\left.{ }^{\circ} 11-19\right)$. The anthropometric, fitness level, and gross motor skill measurements were carried out by physical activity science graduates trained for this study to guarantee standardization. These graduates were responsible for ensuring that the instructions and procedures were understood in each test. 


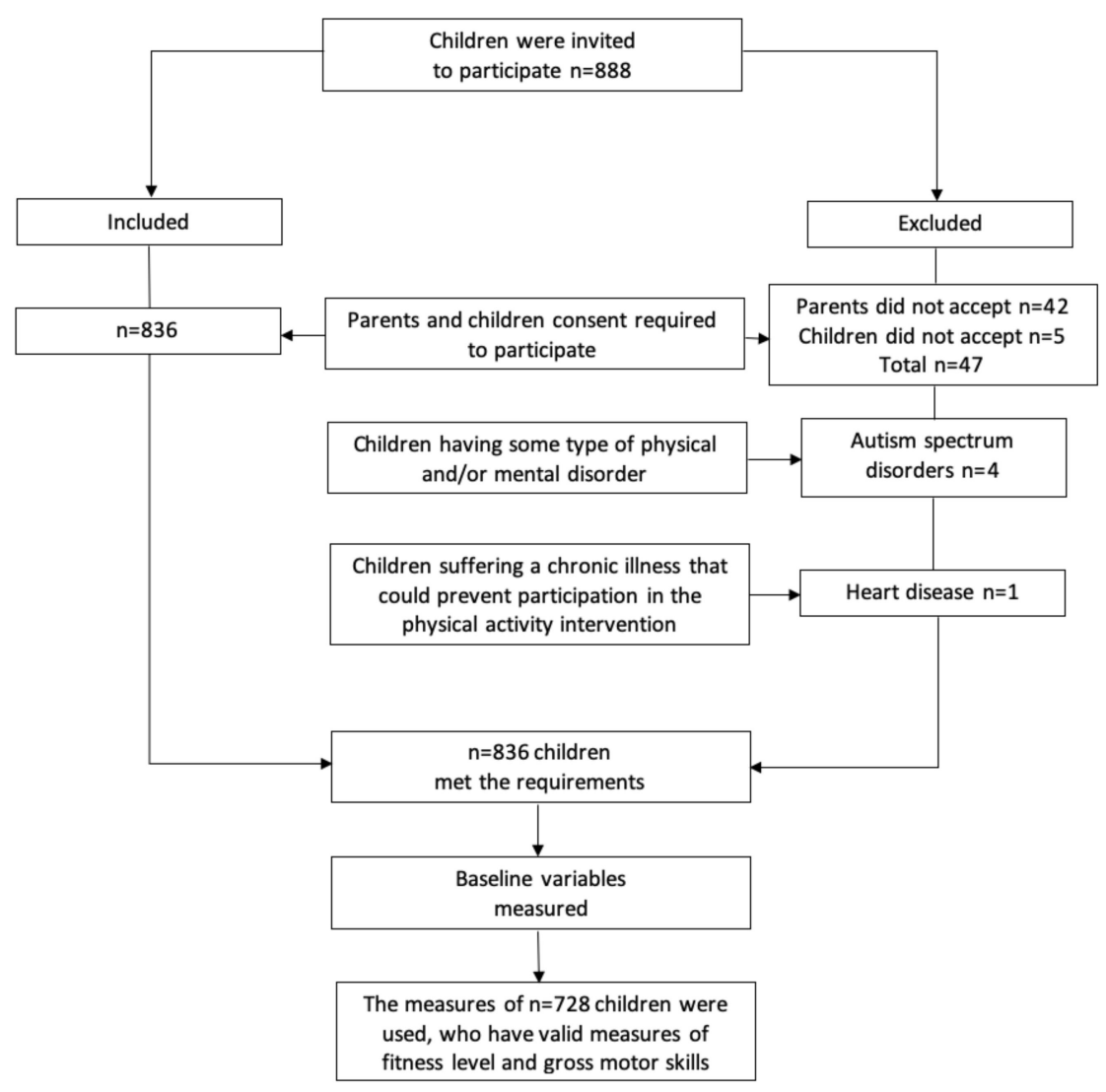

Figure 1. Flow diagram of participant recruitment and exclusion with reasons.

\subsection{Fitness Level}

Cardiorespiratory fitness was assessed using the 20-m shuttle run test, which provides valid and reliable information on maximum aerobic capacity in children [30]. Estimated maximum oxygen volume value (VO2max) was calculated by using the preschool-adapted 20-m shuttle-run (PREFIT) formula [31]. The children were encouraged to run as long as possible during the test, and the last half of the stage reached by the participants was recorded. Muscle strength was assessed using the standing long jump test for lower limb strength; the children were asked to jump horizontally to reach the maximum distance and the result was recorded in $\mathrm{cm}$ as the best value of the three attempts made. For upper limb strength, handgrip dynamometry (Takei 5401) was used, two attempts were made for each hand, the highest values were recorded in $\mathrm{kg}$. Speed/agility was measured using the $4 \times 10 \mathrm{~m}$ shuttle run, two attempts were made with a 5-min rest between them and the lowest value of the two was recorded in seconds. Flexibility was measured with the Sit and Reach test, the result was recorded in $\mathrm{cm}$ as the best value of the two attempts made. These evaluation procedures are part of batteries used to measure fitness level in children [32,33], and provide valid and reliable information [30,32,34,35]. The brief definition of each test is given in Supplementary Materials.

\subsection{Gross Motor Skills}

The validated Spanish version of the second edition of the Movement Assessment Battery for Children (MABC-2) [36], which has proven to be an instrument with adequate psychometric properties of reliability ( $\alpha$ de Cronbach $>0.60 ; \kappa=1$; CCI $=0.85-0.99$ ) [37], was used to measure gross motor skills. This tool was developed to be used in clinical and educational settings. The version with the 4-6 years age range was used. The motor tasks used to assess gross motor skills were: catching and aiming ( 2 tests: catching a beanbag and throwing a beanbag onto a mat), and static and dynamic balance (3 tests: balance for each leg—static balance; tip-toe walking and jumping on a mat-dynamic balance). 


\subsection{Data Analysis}

The adjustment to normal distribution of the different variables was evaluated both by graphs and by the Kolmogorov-Smirnov test. Fitness levels and gross motor skills by age (years) and gender are presented with their mean and standard deviations. Since all variables had a normal distribution, we used parametric tests in the analysis.

Gender differences in fitness levels and gross motor skills were tested using the Student's t statistic (for independent samples).

In order to establish the influence of gender, age in months, height, weight, and body mass index (BMI) on fitness level and gross motor skills, a linear regression analysis was performed adding these variables as fixed factors [38-40].

The ANCOVA model was used to assess differences in fitness level and gross motor skills, controlling for age in months and BMI, by gender.

The percentile values (P10, P20, P30, P40, P50, P60, P70, P80, P90, and P100) were calculated by age and gender for each fitness level and gross motor skills test. Statistical significance was assumed for $p \leq 0.05$. The IBM SPSS Statistics version 25 was used for the data analysis.

\section{Results}

The final sample for this analysis included 728 children (332 boys and 396 girls). The mean weight, height, and BMI values for each age (4, 5, and 6 years old) and gender ranged from 20.0 to $27.1 \mathrm{~kg}$, from 106.5 to $120.3 \mathrm{~cm}$, and from 17.1 to $19.1 \mathrm{~kg} / \mathrm{m}^{2}$, respectively (Table 1 ).

Table 1. Anthropometric characteristics (Mean \pm SD), by age and gender.

\begin{tabular}{|c|c|c|c|}
\hline Age (years) & Weight (kg) & Height (cm) & BMI $\left(\mathrm{kg} / \mathrm{m}^{2}\right)$ \\
\hline \multicolumn{4}{|l|}{ Boys (332) } \\
\hline $4(54)$ & $20.0 \pm 3.1$ & $107.7 \pm 2.7$ & $17.1 \pm 2.1$ \\
\hline $5(112)$ & $23.6 \pm 4.3$ & $114.5 \pm 4.5$ & $17.9 \pm 2.4$ \\
\hline $6(166)$ & $27.0 \pm 5.3$ & $120.3 \pm 5.6$ & $18.5 \pm 2.6$ \\
\hline \multicolumn{4}{|l|}{ Girls (396) } \\
\hline 4 (92) & $20.0 \pm 3$ & $106.5 \pm 5.2$ & $17.5 \pm 1.5$ \\
\hline $5(126)$ & $22.5 \pm 3.8$ & $111.6 \pm 4.7$ & $18.0 \pm 2.4$ \\
\hline $6(178)$ & $27.1 \pm 5.5$ & $118.8 \pm 5.7$ & $19.1 \pm 2.9$ \\
\hline
\end{tabular}

BMI, body mass index (calculated as weight in $\mathrm{kg}$ divided by height in $\mathrm{m}^{2}$ ).

For fitness level, for unadjusted and adjusted values for age and BMI, boys showed better values than girls in the jump ( $p$ from $<0.00$ to 0.00 ), right and left dynamometry ( $p$ from $<0.00$ to 0.12 ), dominant hand dynamometry ( $p$ from $<0.00$ to 0.00 ), no dominant hand dynamometry ( $p$ from $<0.00$ to 0.00 ), and speed/agility tests ( $p$ from 0.01 to 0.72 ), being all statistically significant except left dynamometry at 5 years and speed/agility at 5 and 6 years. Moreover, girls showed significantly higher values than boys in flexibility at all ages ( $p$ from $<0.00$ to 0.00 ). Finally, differences in estimated VO2max were significant for boys at 5 years $(p=0.00)$ and for girls at 6 years $(p=0.04)$ (Table 2).

Table 2. Fitness level values (Mean $\pm \mathrm{SD}$ ), by age and gender.

\begin{tabular}{ccccc}
\hline \multicolumn{4}{c}{ Fitness Level } \\
\hline & Boys $(\boldsymbol{n}=\mathbf{3 3 2})$ & Girls $(\boldsymbol{n}=\mathbf{3 9 6})$ & $\boldsymbol{p}$ \\
\hline Lower limb strength & & & \\
Long jump $(\mathrm{cm})$ & & & \\
4 & Unadjusted & $76.6 \pm 23.5$ & $65.7 \pm 18.9$ & $\mathbf{0 . 0 0}$ \\
& Adjusted & $76.7 \pm 23.5$ & $65.2 \pm 18.7$ & $\mathbf{0 . 0 0}$ \\
5 & Unadjusted & $88.9 \pm 21.5$ & $79.4 \pm 14.7$ & $\mathbf{0 . 0 0}$ \\
& Adjusted & $88.6 \pm 21$ & $79.9 \pm 14.5$ & $\mathbf{0 . 0 0}$ \\
\hline
\end{tabular}


Table 2. Cont.

\begin{tabular}{|c|c|c|c|c|}
\hline \multicolumn{5}{|c|}{ Fitness Level } \\
\hline & & Boys $(n=332)$ & Girls $(n=396)$ & $p$ \\
\hline \multirow[t]{2}{*}{6} & Unadjusted & $96.5 \pm 24.4$ & $86.2 \pm 18.3$ & 0.00 \\
\hline & Adjusted & $96.1 \pm 24$ & $86 \pm 14.7$ & 0.00 \\
\hline \multicolumn{5}{|c|}{ Upper limb strength } \\
\hline \multicolumn{5}{|c|}{ Right Dynam (kg) } \\
\hline \multirow[t]{2}{*}{4} & Unadjusted & $6.1 \pm 1.6$ & $5.2 \pm 2.1$ & 0.00 \\
\hline & Adjusted & $6 \pm 1.4$ & $5.3 \pm 2$ & 0.00 \\
\hline \multirow[t]{2}{*}{5} & Unadjusted & $7.9 \pm 1.6$ & $6.7 \pm 1.3$ & 0.00 \\
\hline & Adjusted & $8.1 \pm 1.7$ & $6.9 \pm 1.1$ & 0.00 \\
\hline \multirow[t]{2}{*}{6} & Unadjusted & $9 \pm 2.1$ & $8.1 \pm 2.1$ & 0.00 \\
\hline & Adjusted & $9.1 \pm 2.2$ & $8.4 \pm 2.2$ & 0.01 \\
\hline \multicolumn{5}{|c|}{ Left Dynam (kg) } \\
\hline \multirow[t]{2}{*}{4} & Unadjusted & $6.1 \pm 1.5$ & $5.1 \pm 2.1$ & 0.00 \\
\hline & Adjusted & $6.3 \pm 1.7$ & $5.3 \pm 2.1$ & 0.00 \\
\hline \multirow[t]{2}{*}{5} & Unadjusted & $7.1 \pm 1.9$ & $6.7 \pm 1.7$ & 0.12 \\
\hline & Adjusted & $7.2 \pm 1.7$ & $6.9 \pm 1.5$ & 0.14 \\
\hline \multirow[t]{2}{*}{6} & Unadjusted & $9.2 \pm 2.2$ & $7.9 \pm 2$ & 0.00 \\
\hline & Adjusted & $9.5 \pm 2$ & $8.1 \pm 1.7$ & 0.01 \\
\hline \multicolumn{5}{|c|}{ Dominant hand (kg) } \\
\hline \multirow[t]{2}{*}{4} & Unadjusted & $6.3 \pm 1.4$ & $5.3 \pm 2$ & 0.00 \\
\hline & Adjusted & $6.5 \pm 1.7$ & $5.7 \pm 2.3$ & 0.00 \\
\hline \multirow[t]{2}{*}{5} & Unadjusted & $7.9 \pm 1.4$ & $6.9 \pm 1.8$ & 0.00 \\
\hline & Adjusted & $8.1 \pm 1.6$ & $7.1 \pm 2$ & 0.00 \\
\hline \multirow[t]{2}{*}{6} & Unadjusted & $9.2 \pm 1.4$ & $8.2 \pm 2.1$ & 0.00 \\
\hline & Adjusted & $9.8 \pm 1.5$ & $9.1 \pm 3.2$ & 0.00 \\
\hline \multicolumn{5}{|c|}{ No dominant hand (kg) } \\
\hline \multirow[t]{2}{*}{4} & Unadjusted & $5.4 \pm 1.3$ & $4.7 \pm 1.8$ & 0.00 \\
\hline & Adjusted & $5.4 \pm 1.5$ & $4.9 \pm 1.6$ & 0.00 \\
\hline \multirow[t]{2}{*}{5} & Unadjusted & $6.8 \pm 1.6$ & $6.4 \pm 1.6$ & 0.00 \\
\hline & Adjusted & $7.1 \pm 1.9$ & $6.9 \pm 2$ & 0.00 \\
\hline 6 & Unadjusted & $8.5 \pm 1.2$ & $7.1 \pm 1.7$ & 0.00 \\
\hline & Adjusted & $9 \pm 1.6$ & $7.7 \pm 1.1$ & 0.00 \\
\hline Flexibility & & & & \\
\hline Sit and reach & & & & \\
\hline 4 & Unadjusted & $27.2 \pm 3.4$ & $29.1 \pm 4.1$ & 0.00 \\
\hline & Adjusted & $27.4 \pm 3.5$ & $29.5 \pm 4$ & 0.00 \\
\hline 5 & Unadjusted & $31.1 \pm 4$ & $32.6 \pm 4.6$ & 0.00 \\
\hline & Adjusted & $31.4 \pm 4.2$ & $33.1 \pm 4$ & 0.00 \\
\hline 6 & Unadjusted & $29.7 \pm 5.4$ & $32.4 \pm 5.2$ & 0.00 \\
\hline & Adjusted & $29.9 \pm 5.2$ & $33 \pm 4.9$ & 0.00 \\
\hline Speed/Agili & & & & \\
\hline $4 \times 10(\mathrm{sec})$ & & & & \\
\hline 4 & Unadjusted & $17.4 \pm 1.5$ & $18.2 \pm 1.8$ & 0.01 \\
\hline & Adjusted & $17.3 \pm 1.2$ & $18 \pm 1.5$ & 0.00 \\
\hline 5 & Unadjusted & $16.8 \pm 2.4$ & $17.4 \pm 2.1$ & 0.06 \\
\hline & Adjusted & $16.5 \pm 2$ & $17.2 \pm 2$ & 0.06 \\
\hline 6 & Unadjusted & $16.3 \pm 1.8$ & $16.3 \pm 1.3$ & 0.72 \\
\hline & Adjusted & $15.9 \pm 2$ & $16 \pm 1.8$ & 0.72 \\
\hline Cardiorespi & & & & \\
\hline Course nave & & & & \\
\hline 4 & Unadjusted & $1.2 \pm 0.6$ & $1.3 \pm 0.6$ & 0.59 \\
\hline & Adjusted & $1.1 \pm 0.8$ & $1.1 \pm 0.4$ & 0.59 \\
\hline 5 & Unadjusted & $1.7 \pm 0.6$ & $1.4 \pm 0.6$ & 0.00 \\
\hline & Adjusted & $1.8 \pm 0.6$ & $1.6 \pm 0.6$ & 0.00 \\
\hline 6 & Unadjusted & $1.8 \pm 0.9$ & $2 \pm 0.8$ & 0.10 \\
\hline & Adjusted & $2 \pm 0.6$ & $2.2 \pm 0.8$ & 0.10 \\
\hline
\end{tabular}


Table 2. Cont.

\begin{tabular}{ccccc}
\hline \multicolumn{4}{c}{ Fitness Level } \\
\hline & Boys $(\boldsymbol{n}=\mathbf{3 3 2})$ & Girls $(\boldsymbol{n}=\mathbf{3 9 6})$ & $\boldsymbol{p}$ \\
\hline VO2max $(\mathrm{mL} / \mathrm{kg} / \mathrm{min})$ & & & \\
4 & Unadjusted & $47.1 \pm 1.3$ & $47 \pm 1.4$ & 0.66 \\
& Adjusted & $47.5 \pm 1.5$ & $47.6 \pm 1.8$ & 0.66 \\
5 & Unadjusted & $47.8 \pm 1.4$ & $47.2 \pm 1.3$ & $\mathbf{0 . 0 0}$ \\
& Adjusted & $47.5 \pm 1.6$ & $47 \pm 1.1$ & $\mathbf{0 . 0 0}$ \\
6 & Unadjusted & $48 \pm 2$ & $48.4 \pm 1.8$ & 0.04 \\
& Adjusted & $48.1 \pm 2.2$ & $48.3 \pm 1.9$ & 0.04 \\
\hline
\end{tabular}

$¥$ Less time (in sec) indicates better fitness level, ${ }^{\ddagger} 1$ stage $=1 \mathrm{~min}$. The values in bold indicate a statistical significance for $p<0.05$.

Regarding gross motor skills, for unadjusted and adjusted values for age and BMI, boys obtained better values than girls for the catching and aiming tests, showing statistically significant differences for the catching at $4(p=0.02)$ and $5(p=0.04)$ years and for the aiming at 5 years $(p=0.00)$. For both static and dynamic balance, girls obtained higher values than boys ( $p$ from $<0.00$ to 0.92$)$, being all statistically significant except right balance at 4 years, left balance at 4 and 5 years and tip-toes at 5 years (Table 3).

Table 3. Gross motor skills values (Mean \pm SD), by age and gender.

\begin{tabular}{|c|c|c|c|c|}
\hline \multicolumn{5}{|c|}{ Gross Motor Skills } \\
\hline & & Boys $(n=332)$ & Girls $(n=396)$ & $p$ \\
\hline \multicolumn{5}{|c|}{ Catching and aiming } \\
\hline \multicolumn{5}{|c|}{ Catching (number) } \\
\hline \multirow[t]{2}{*}{4} & Unadjusted & $7.1 \pm 2.7$ & $6 \pm 2.9$ & 0.02 \\
\hline & Adjusted & $7 \pm 2.5$ & $5.8 \pm 2.4$ & 0.03 \\
\hline \multirow[t]{2}{*}{5} & Unadjusted & $8.5 \pm 2.3$ & $7.8 \pm 2.4$ & 0.04 \\
\hline & Adjusted & $8.6 \pm 2.5$ & $7.9 \pm 2.2$ & 0.02 \\
\hline \multirow[t]{2}{*}{6} & Unadjusted & $8.9 \pm 2$ & $8.9 \pm 1.7$ & 0.87 \\
\hline & Adjusted & $9.1 \pm 1.6$ & $9 \pm 2.1$ & 0.85 \\
\hline \multicolumn{5}{|c|}{ Aiming (number) } \\
\hline \multirow[t]{2}{*}{4} & Unadjusted & $3.8 \pm 2.6$ & $4.1 \pm 2.6$ & 0.49 \\
\hline & Adjusted & $3.7 \pm 2.4$ & $4.2 \pm 2.2$ & 0.45 \\
\hline \multirow[t]{2}{*}{5} & Unadjusted & $4.5 \pm 2.1$ & $3.7 \pm 2.3$ & 0.00 \\
\hline & Adjusted & $4.7 \pm 2.3$ & $3.8 \pm 2$ & 0.00 \\
\hline \multirow[t]{2}{*}{6} & Unadjusted & $5.2 \pm 2.2$ & $5.1 \pm 3.4$ & 0.73 \\
\hline & Adjusted & $5.4 \pm 2.5$ & $5.3 \pm 3.8$ & 0.69 \\
\hline \multicolumn{5}{|l|}{ Balance } \\
\hline \multicolumn{5}{|c|}{ Right balance (sec) } \\
\hline \multirow[t]{2}{*}{4} & Unadjusted & $10.3 \pm 6,5$ & $15,6 \pm 10.3$ & 0.00 \\
\hline & Adjusted & $10.1 \pm 5.9$ & $16 \pm 9.9$ & 0.00 \\
\hline \multirow[t]{2}{*}{5} & Unadjusted & $19 \pm 10$ & $16.7 \pm 9.2$ & 0.06 \\
\hline & Adjusted & $18.6 \pm 10$ & $17.1 \pm 9$ & 0.06 \\
\hline \multirow[t]{2}{*}{6} & Unadjusted & $18.5 \pm 10.4$ & $24,9 \pm 8.1$ & 0.00 \\
\hline & Adjusted & $18.9 \pm 10$ & $25 \pm 7$ & 0.01 \\
\hline \multicolumn{5}{|c|}{ Left balance (sec) } \\
\hline \multirow[t]{2}{*}{4} & Unadjusted & $12.7 \pm 9.7$ & $12.8 \pm 9$ & 0.92 \\
\hline & Adjusted & $12.9 \pm 9.3$ & $13 \pm 8.9$ & 0.88 \\
\hline \multirow[t]{2}{*}{5} & Unadjusted & $17.2 \pm 10.7$ & $19.1 \pm 9.8$ & 0.16 \\
\hline & Adjusted & $17.5 \pm 9.6$ & $19.2 \pm 9$ & 0.19 \\
\hline \multirow[t]{2}{*}{6} & Unadjusted & $20.2 \pm 10$ & $23.8 \pm 8.5$ & 0.00 \\
\hline & Adjusted & $20.9 \pm 10$ & $24.1 \pm 8$ & 0.00 \\
\hline
\end{tabular}


Table 3. Cont.

\begin{tabular}{|c|c|c|c|c|}
\hline \multicolumn{5}{|c|}{ Gross Motor Skills } \\
\hline & & Boys $(n=332)$ & Girls $(n=396)$ & $p$ \\
\hline \multicolumn{5}{|c|}{ Dominant leg (sec) } \\
\hline \multirow[t]{2}{*}{4} & Unadjusted & $10.4 \pm 5.8$ & $16.6 \pm 9$ & 0.00 \\
\hline & Adjusted & $10.2 \pm 5.9$ & $16.7 \pm 8.1$ & 0.00 \\
\hline \multirow[t]{2}{*}{5} & Unadjusted & $19.3 \pm 9.4$ & $17.1 \pm 8$ & 0.00 \\
\hline & Adjusted & $19.5 \pm 9$ & $17.6 \pm 8.2$ & 0.00 \\
\hline \multirow[t]{2}{*}{6} & Unadjusted & $19.4 \pm 9.9$ & $25.9 \pm 7.1$ & 0.00 \\
\hline & Adjusted & $21.6 \pm 9.1$ & $26 \pm 7$ & 0.00 \\
\hline \multicolumn{5}{|c|}{ No dominant leg (sec) } \\
\hline \multirow[t]{2}{*}{4} & Unadjusted & $11.5 \pm 9$ & $12.6 \pm 8.8$ & 0.00 \\
\hline & Adjusted & $11.4 \pm 8.8$ & $12.7 \pm 8.7$ & 0.01 \\
\hline \multirow[t]{2}{*}{5} & Unadjusted & $17.1 \pm 9.2$ & $18.8 \pm 9$ & 0.00 \\
\hline & Adjusted & $17.3 \pm 9.6$ & $18.9 \pm 9.1$ & 0.00 \\
\hline \multirow[t]{2}{*}{6} & Unadjusted & $17.2 \pm 8.8$ & $24 \pm 8.2$ & 0.00 \\
\hline & Adjusted & $17.6 \pm 8.1$ & $24.2 \pm 8.3$ & 0.00 \\
\hline \multicolumn{5}{|c|}{ Tip-toes (steps) } \\
\hline \multirow[t]{2}{*}{4} & Unadjusted & $11.3 \pm 5.4$ & $13.5 \pm 3.2$ & 0.00 \\
\hline & Adjusted & $11.1 \pm 5$ & $13.1 \pm 3$ & 0.00 \\
\hline \multirow[t]{2}{*}{5} & Unadjusted & $13.1 \pm 3.9$ & $13.7 \pm 3.3$ & 0.23 \\
\hline & Adjusted & $13.5 \pm 4.1$ & $13.9 \pm 2.5$ & 0.21 \\
\hline \multirow[t]{2}{*}{6} & Unadjusted & $13.8 \pm 3.1$ & $14.7 \pm 1.2$ & 0.00 \\
\hline & Adjusted & $14 \pm 3$ & $14.9 \pm 1.5$ & 0.00 \\
\hline \multicolumn{5}{|c|}{ Floor mat (jumps) } \\
\hline \multirow[t]{2}{*}{4} & Unadjusted & $4.4 \pm 0.7$ & $4.9 \pm 0.3$ & 0.00 \\
\hline & Adjusted & $4.3 \pm 0.8$ & $4.8 \pm 0.2$ & 0.00 \\
\hline \multirow[t]{2}{*}{5} & Unadjusted & $4.7 \pm 0.6$ & $4.9 \pm 0.2$ & 0.02 \\
\hline & Adjusted & $4.8 \pm 0.5$ & $4.9 \pm 0.4$ & 0.01 \\
\hline \multirow[t]{2}{*}{6} & Unadjusted & $4.7 \pm 3.1$ & $4.9 \pm 0.3$ & 0.00 \\
\hline & Adjusted & $4.8 \pm 3.3$ & $4.9 \pm 0.5$ & 0.00 \\
\hline
\end{tabular}

The values in bold indicate a statistical significance for $p<0.05$.

Age in months, height, weight, and BMI were strongly associated with fitness level and gross motor skills in both boys and girls (Table 4). 
Table 4. Linear regression model for fitness level and gross motor skills outcomes adjusted by gender, age in months, height, weight, and BMI.

\begin{tabular}{|c|c|c|c|c|c|}
\hline Variable & Gender & $\mathbf{R}^{2}$ & Estimate & SE & $p$ \\
\hline \multirow{2}{*}{ Long jump } & Boys & $62 \%$ & 5.6 & 2.4 & 0.02 \\
\hline & Girls & $56 \%$ & 8.9 & 1.4 & 0.00 \\
\hline \multirow{2}{*}{ Right dinamometry } & Boys & $73 \%$ & 0.59 & 0.18 & 0.00 \\
\hline & Girls & $71 \%$ & 0.46 & 0.15 & 0.00 \\
\hline \multirow{2}{*}{ Left dinamometry } & Boys & $54 \%$ & 1.2 & 0.21 & 0.00 \\
\hline & Girls & $58 \%$ & 0.45 & 0.15 & 0.00 \\
\hline \multirow{2}{*}{ Dominant hand dinamometry } & Boys & $75 \%$ & 0.36 & 0.19 & 0.02 \\
\hline & Girls & $75 \%$ & 0.23 & 0.11 & 0.00 \\
\hline \multirow{2}{*}{ No dominant hand dinamometry } & Boys & $66 \%$ & 0.31 & 0.15 & 0.01 \\
\hline & Girls & $67 \%$ & 0.27 & 0.15 & 0.00 \\
\hline \multirow{2}{*}{ Sit and reach } & Boys & $56 \%$ & 1.3 & 0.22 & 0.05 \\
\hline & Girls & $57 \%$ & 0.78 & 0.42 & 0.08 \\
\hline \multirow{2}{*}{$4 \times 10 \mathrm{~m}$} & Boys & $73 \%$ & 0.13 & 0.09 & 0.05 \\
\hline & Girls & $71 \%$ & 0.56 & 0.14 & 0.00 \\
\hline \multirow{2}{*}{$20-\mathrm{m}$ shuttle run test } & Boys & $88 \%$ & 0.15 & 0.08 & 0.05 \\
\hline & Girls & $87 \%$ & 0.35 & 0.06 & 0.00 \\
\hline \multirow{2}{*}{ Estimate VO2max } & Boys & $86 \%$ & 0.22 & 0.18 & 0.00 \\
\hline & Girls & $86 \%$ & 0.67 & 0.14 & 0.00 \\
\hline \multirow{2}{*}{ Catching } & Boys & $59 \%$ & 0.41 & 0.23 & 0.02 \\
\hline & Girls & $61 \%$ & 1.3 & 0.2 & 0.00 \\
\hline \multirow{2}{*}{ Aiming } & Boys & $58 \%$ & 0.21 & 0.11 & 0.00 \\
\hline & Girls & $66 \%$ & 0.4 & 0.26 & 0.01 \\
\hline \multirow{2}{*}{ Right balance } & Boys & $70 \%$ & 1.6 & 1 & 0.02 \\
\hline & Girls & $68 \%$ & 0.36 & 0.16 & 0.00 \\
\hline \multirow{2}{*}{ Leght balance } & Boys & $71 \%$ & 0.43 & 0.23 & 0.00 \\
\hline & Girls & $74 \%$ & 0.47 & 0.25 & 0.05 \\
\hline \multirow{2}{*}{ Balance dominant leg } & Boys & $65 \%$ & 0.45 & 0.12 & 0.00 \\
\hline & Girls & $61 \%$ & 0.39 & 0.15 & 0.00 \\
\hline \multirow{2}{*}{ Balance no dominant leg } & Boys & $67 \%$ & 0.33 & 0.23 & 0.04 \\
\hline & Girls & $67 \%$ & 0.31 & 0.24 & 0.05 \\
\hline \multirow{2}{*}{ Tip-toes } & Boys & $59 \%$ & 0.35 & 0.12 & 0.05 \\
\hline & Girls & $62 \%$ & 0.68 & 0.22 & 0.03 \\
\hline \multirow{2}{*}{ Floor mat } & Boys & $66 \%$ & 0.3 & 0.1 & 0.05 \\
\hline & Girls & $63 \%$ & 0.02 & 0.01 & 0.00 \\
\hline
\end{tabular}

The values in bold indicate a statistical significance for $p<0.05$.

Finally, Tables 5 and 6 (girls), and Tables 7 and 8 (boys) show percentile values for each physical fitness and gross motor skills test. Fitness level and gross motor skills were improved with age. 
Table 5. Percentile values for girls in fitness level.

\begin{tabular}{|c|c|c|c|c|c|c|c|c|c|}
\hline Age (Years) & $\begin{array}{l}\text { Long Jump } \\
\text { (cm) }\end{array}$ & $\begin{array}{c}\text { Speed/agility } \\
\text { (Sec) }\end{array}$ & $\begin{array}{c}\text { Right } \\
\text { Dynamometry (kg) }\end{array}$ & $\begin{array}{l}\text { Left Dynamometry } \\
(\mathbf{k g})\end{array}$ & $\begin{array}{c}\text { Dominant Hand } \\
\text { Dynamometry (kg) }\end{array}$ & $\begin{array}{l}\text { No Dominant Hand } \\
\text { Dynamometry (kg) }\end{array}$ & $\begin{array}{l}\text { Flexibility } \\
\quad(\mathrm{cm})\end{array}$ & $\begin{array}{c}\text { 20-m Shuttle Run } \\
\text { Test (Stage) } \ddagger\end{array}$ & $\begin{array}{l}\text { Estimated VO2max } \\
(\mathrm{mL} / \mathrm{kg} / \mathrm{min})\end{array}$ \\
\hline \multicolumn{10}{|l|}{$4(n=92)$} \\
\hline p10 & 42 & 23.5 & 2 & 2 & 2.6 & 2.3 & 23 & 0.4 & 46 \\
\hline p20 & 49.2 & 20.9 & 2.2 & 2.2 & 3.2 & 2.5 & 25.6 & 1 & 46 \\
\hline p30 & 54 & 19.4 & 2.2 & 5 & 3.4 & 2.5 & 27 & 1 & 46 \\
\hline $\mathrm{p} 40$ & 63 & 18.9 & 6 & 5.2 & 6 & 6 & 29 & 1.1 & 46.1 \\
\hline p50 & 67.5 & 18.6 & 6.1 & 5.5 & 6.7 & 6.6 & 29 & 1.2 & 46.9 \\
\hline p60 & 71 & 18.2 & 6.3 & 5.8 & 7.4 & 6.8 & 30 & 1.3 & 46.9 \\
\hline p70 & 78 & 17.1 & 6.8 & 6.2 & 8.3 & 7.4 & 31 & 1.4 & 46.9 \\
\hline p80 & 80 & 16.6 & 7.2 & 6.8 & 8.9 & 7.8 & 32 & 2 & 49 \\
\hline p90 & 90 & 16.3 & 7.4 & 7.9 & 9.3 & 8 & 34 & 2.2 & 49 \\
\hline p100 & 104 & 16.1 & 7.8 & 8.7 & 9.9 & 8.4 & 41 & 3.2 & 53.1 \\
\hline \multicolumn{10}{|l|}{$5(n=126)$} \\
\hline p10 & 60 & 25.1 & 5 & 5.1 & 5.2 & 5.2 & 26.4 & 0.3 & 46 \\
\hline p20 & 69 & 19.9 & 5.2 & 5.6 & 5.7 & 5.4 & 30 & 1 & 46 \\
\hline p30 & 72.1 & 18.7 & 5.6 & 5.8 & 6.3 & 5.7 & 31 & 1.1 & 46 \\
\hline $\mathrm{p} 40$ & 75.8 & 18.1 & 6.2 & 6.2 & 6.7 & 6.4 & 32 & 1.1 & 46.9 \\
\hline p50 & 78 & 17.8 & 6.5 & 6.9 & 7.4 & 6.7 & 33 & 1.2 & 46.9 \\
\hline p60 & 82 & 17.4 & 6.8 & 7.4 & 8.2 & 7.2 & 34 & 1.3 & 46.9 \\
\hline p70 & 86 & 16.4 & 7.3 & 7.7 & 9.1 & 7.8 & 35 & 1.9 & 48.7 \\
\hline p80 & 92 & 16.4 & 8.5 & 8.2 & 9.6 & 8.6 & 36 & 2 & 49 \\
\hline p90 & 100.6 & 15.8 & 9.1 & 8.9 & 10.4 & 9.3 & 37.6 & 2.3 & 49 \\
\hline p100 & 109 & 14.7 & 9.4 & 9.9 & 11 & 10 & 43 & 3.2 & 51.1 \\
\hline \multicolumn{10}{|l|}{$6(n=178)$} \\
\hline p10 & 66 & 20.4 & 5.8 & 5.5 & 5.8 & 5.8 & 25 & 1.1 & 46 \\
\hline p20 & 72 & 18.2 & 5.9 & 6.3 & 6.2 & 5.9 & 28 & 1.3 & 46.9 \\
\hline p30 & 77 & 17.5 & 6.3 & 6.8 & 7 & 6.4 & 29 & 1.4 & 46.9 \\
\hline $\mathrm{p} 40$ & 81 & 16.9 & 7.2 & 7.1 & 7.6 & 7.3 & 31.5 & 2 & 49 \\
\hline p50 & 85 & 16.3 & 7.8 & 7.8 & 8.2 & 7.8 & 33 & 2.1 & 49 \\
\hline p60 & 90 & 15.9 & 9 & 8.1 & 9 & 8.9 & 35 & 2.1 & 49 \\
\hline p70 & 95 & 15.8 & 9.4 & 8.7 & 9.6 & 9.5 & 36 & 2.2 & 49 \\
\hline p80 & 101 & 15.6 & 9.9 & 9.7 & 10.3 & 10.1 & 36 & 2.4 & 49 \\
\hline p90 & 108 & 15.2 & 10.3 & 11.2 & 13.4 & 12.6 & 38 & 3.4 & 51.1 \\
\hline p100 & 140 & 14.8 & 14.1 & 15 & 15.7 & 15 & 45 & 4.4 & 53.1 \\
\hline
\end{tabular}

${ }^{¥}$ Less time (in sec) indicates better fitness level, $\ddagger 1$ stage $=1 \mathrm{~min}$. 
Table 6. Percentile values for girls in gross motor skills.

\begin{tabular}{|c|c|c|c|c|c|c|c|c|}
\hline Age (Years) & $\begin{array}{l}\text { Catching } \\
\text { (Numbers) }\end{array}$ & $\begin{array}{c}\text { Aiming } \\
\text { (Numbers) }\end{array}$ & $\begin{array}{l}\text { Right Balance } \\
\text { (Sec) }\end{array}$ & $\begin{array}{l}\text { Left Balance } \\
\text { (Sec) }\end{array}$ & $\begin{array}{c}\text { Dominant Leg } \\
\text { Balance (Sec) }\end{array}$ & $\begin{array}{l}\text { No Dominant Leg } \\
\text { Balance (Sec) }\end{array}$ & $\begin{array}{c}\text { Tip-Toes } \\
\text { (Steps/Numbers) }\end{array}$ & $\begin{array}{c}\text { Floor Mat } \\
\text { (Jumps) }\end{array}$ \\
\hline \multicolumn{9}{|l|}{$4(n=92)$} \\
\hline p10 & 2 & 1 & 3 & 3 & 5 & 3 & 8 & 0 \\
\hline p20 & 3 & 2 & 4.6 & 5.6 & 7 & 5 & 14.2 & 1 \\
\hline p30 & 4 & 3 & 6 & 6 & 8 & 7 & 15 & 1 \\
\hline p40 & 6 & 3 & 10 & 10 & 14 & 12 & 15 & 2 \\
\hline p50 & 6 & 4 & 15.5 & 15.5 & 16 & 18 & 15 & 3 \\
\hline p 60 & 7 & 4 & 21 & 21 & 22 & 21 & 15 & 4 \\
\hline p70 & 9 & 5 & 24 & 24 & 28 & 24 & 15 & 4 \\
\hline p80 & 9 & 6 & 26 & 26 & 30 & 27 & 15 & 5 \\
\hline p90 & 10 & 9 & 30 & 30 & 30 & 30 & 15 & 5 \\
\hline p100 & 10 & 10 & 30 & 30 & 30 & 30 & 15 & 5 \\
\hline \multicolumn{9}{|l|}{$5(n=126)$} \\
\hline p10 & 4.7 & 1 & 6.7 & 6 & 8 & 6 & 5 & 1 \\
\hline p20 & 5 & 2 & 8 & 8 & 12 & 8 & 15 & 1 \\
\hline p30 & 7 & 2 & 10 & 11 & 17 & 14 & 15 & 2 \\
\hline p 40 & 8 & 3 & 11.2 & 14 & 21 & 19 & 15 & 3 \\
\hline p50 & 9 & 3 & 14 & 20 & 24 & 22 & 15 & 3 \\
\hline p60 & 9 & 4 & 16.2 & 25.2 & 27 & 26 & 15 & 4 \\
\hline p70 & 10 & 5 & 24.7 & 30 & 30 & 28 & 15 & 5 \\
\hline p80 & 10 & 6 & 30 & 30 & 30 & 30 & 15 & 5 \\
\hline p90 & 10 & 7 & 30 & 30 & 30 & 30 & 15 & 5 \\
\hline p100 & 10 & 9 & 30 & 30 & 30 & 30 & 15 & 5 \\
\hline \multicolumn{9}{|l|}{$6(n=178)$} \\
\hline p10 & 7 & 2 & 12 & 10 & 15 & 9 & 15 & 1 \\
\hline p20 & 8 & 3 & 17 & 15 & 20 & 12 & 15 & 2 \\
\hline p30 & 9 & 4 & 25 & 19 & 26 & 20 & 15 & 3 \\
\hline $\mathrm{p} 40$ & 10 & 4 & 30 & 26 & 30 & 30 & 15 & 3 \\
\hline p50 & 10 & 5 & 30 & 30 & 30 & 30 & 15 & 4 \\
\hline p60 & 10 & 5 & 30 & 30 & 30 & 30 & 15 & 5 \\
\hline p70 & 10 & 6 & 30 & 30 & 30 & 30 & 15 & 5 \\
\hline p80 & 10 & 7 & 30 & 30 & 30 & 30 & 15 & 5 \\
\hline p90 & 10 & 8 & 30 & 30 & 30 & 30 & 15 & 5 \\
\hline p100 & 10 & 10 & 30 & 30 & 30 & 30 & 15 & 5 \\
\hline
\end{tabular}


Table 7. Percentile values for boys in fitness level.

\begin{tabular}{|c|c|c|c|c|c|c|c|c|c|}
\hline Age (Years) & $\begin{array}{l}\text { Long Jump } \\
\text { (cm) }\end{array}$ & $\begin{array}{l}\text { Speed/Agility } \\
\text { (Sec) }\end{array}$ & $\begin{array}{c}\text { Right } \\
\text { Dynamometry (kg) }\end{array}$ & $\begin{array}{l}\text { Left Dynamometry } \\
(\mathbf{k g})\end{array}$ & $\begin{array}{c}\text { Dominant Hand } \\
\text { Dynamometry (kg) }\end{array}$ & $\begin{array}{l}\text { No Dominant Hand } \\
\text { Dynamometry (kg) }\end{array}$ & $\begin{array}{l}\text { Flexibility } \\
\quad(\mathrm{cm})\end{array}$ & $\begin{array}{c}\text { 20-m Shuttle Run } \\
\text { Test (stage) } \ddagger\end{array}$ & $\begin{array}{l}\text { Estimated VO2max } \\
(\mathrm{mL} / \mathrm{kg} / \mathrm{min})\end{array}$ \\
\hline \multicolumn{10}{|l|}{$4(n=54)$} \\
\hline p10 & 32 & 21 & 5 & 5 & 6 & 4.8 & 23 & 0.4 & 46 \\
\hline p20 & 58 & 19.4 & 5.2 & 5 & 6.6 & 5.3 & 24 & 1 & 46 \\
\hline p30 & 69 & 18.8 & 5.3 & 5.6 & 7 & 5.9 & 26 & 1 & 46 \\
\hline $\mathrm{p} 40$ & 78 & 18.4 & 5.5 & 5.8 & 7.3 & 6.4 & 26 & 1 & 46.9 \\
\hline p50 & 83 & 17.8 & 5.6 & 6 & 7.8 & 6.6 & 27 & 1.1 & 46.9 \\
\hline p60 & 90 & 17.2 & 6.6 & 6.6 & 8.2 & 6.9 & 28 & 1.3 & 46.9 \\
\hline p70 & 93 & 16.5 & 7.2 & 6.8 & 8.5 & 7.1 & 28 & 1.4 & 46.9 \\
\hline p80 & 95 & 16.3 & 7.4 & 7.5 & 8.9 & 7.5 & 30 & 2 & 49 \\
\hline p90 & 97 & 16.2 & 8.4 & 7.8 & 9.1 & 8.1 & 32 & 2 & 49 \\
\hline p100 & 111 & 15.4 & 8.8 & 8.7 & 9.5 & 8.8 & 34 & 3.2 & 51.1 \\
\hline \multicolumn{10}{|l|}{$5(n=112)$} \\
\hline p10 & 62 & 27.9 & 5.3 & 5.2 & 5.7 & 5.5 & 26.5 & 1 & 46 \\
\hline p20 & 74.2 & 18.5 & 6.5 & 5.5 & 6.7 & 5.8 & 27 & 1.1 & 46 \\
\hline p30 & 80 & 18.2 & 7.1 & 6 & 7.3 & 6.4 & 29 & 1.3 & 46.9 \\
\hline $\mathrm{p} 40$ & 88 & 17.3 & 7.7 & 6.6 & 7.7 & 6.9 & 30 & 1.4 & 46.9 \\
\hline p50 & 91 & 16.8 & 7.9 & 6.8 & 8.3 & 7.2 & 31 & 2 & 49 \\
\hline p60 & 97 & 16.5 & 8.5 & 7.7 & 9 & 7.7 & 32 & 2 & 49 \\
\hline p70 & 100 & 15.9 & 9.1 & 7.8 & 9.8 & 8.2 & 34 & 2.2 & 49 \\
\hline p80 & 106 & 15.7 & 9.6 & 9.1 & 10.4 & 8.9 & 34 & 2.3 & 49 \\
\hline p90 & 116 & 15.3 & 10 & 9.9 & 10.9 & 9.4 & 36 & 2.5 & 49 \\
\hline p100 & 140 & 14.4 & 10.6 & 11.2 & 12.1 & 10.2 & 41 & 3 & 51.1 \\
\hline \multicolumn{10}{|l|}{$6(n=166)$} \\
\hline p10 & 72.1 & 25.2 & 6.6 & 6.2 & 6.8 & 6.7 & 23 & 1 & 46 \\
\hline p20 & 80 & 18.2 & 7.4 & 7.2 & 7.5 & 7.3 & 27 & 1.1 & 46 \\
\hline p30 & 85 & 17.2 & 8.1 & 8.2 & 8.3 & 7.9 & 28 & 1.3 & 46.9 \\
\hline $\mathrm{p} 40$ & 91.8 & 16.7 & 8.5 & 8.6 & 8.9 & 8.4 & 29 & 1.4 & 46.9 \\
\hline p50 & 97 & 16.4 & 8.9 & 9.4 & 9.5 & 9 & 30 & 1.5 & 46.9 \\
\hline p60 & 101.4 & 16.2 & 9.6 & 9.6 & 10 & 9.4 & 31 & 2.1 & 49 \\
\hline p70 & 105.9 & 15.7 & 10.3 & 9.9 & 10.4 & 9.9 & 32 & 2.2 & 49 \\
\hline p80 & 110 & 15.4 & 10.6 & 10.6 & 11.1 & 10 & 34 & 2.4 & 49 \\
\hline p90 & 126.3 & 14.9 & 11.4 & 12.1 & 14.8 & 12 & 36 & 3.2 & 51.1 \\
\hline p100 & 220 & 14.3 & 14.7 & 16.7 & 17 & 14.5 & 42 & 4.2 & 53.1 \\
\hline
\end{tabular}

${ }^{¥}$ Less time (in sec) indicates better fitness level, $\ddagger 1$ stage $=1 \mathrm{~min}$ 
Table 8. Percentile values for boys in gross motor skills.

\begin{tabular}{|c|c|c|c|c|c|c|c|c|}
\hline Age (Years) & $\begin{array}{l}\text { Catching } \\
\text { (Number) }\end{array}$ & $\begin{array}{l}\text { Aiming } \\
\text { (Number) }\end{array}$ & $\begin{array}{l}\text { Right Balance } \\
\text { (Sec) }\end{array}$ & $\begin{array}{l}\text { Left Balance } \\
\text { (Sec) }\end{array}$ & $\begin{array}{l}\text { Dominant Leg } \\
\text { Balance (Sec) }\end{array}$ & $\begin{array}{l}\text { No Dominant Leg } \\
\text { Balance (Sec) }\end{array}$ & $\begin{array}{c}\text { Tip-Toes } \\
\text { (Steps/Numbers) }\end{array}$ & $\begin{array}{c}\text { Floor Mat } \\
\text { (Jumps) }\end{array}$ \\
\hline \multicolumn{9}{|l|}{$4(n=54)$} \\
\hline p10 & 3 & 1 & 2 & 3 & 4 & 2 & 8 & 0 \\
\hline p20 & 5 & 2 & 5 & 5 & 7 & 5 & 14.2 & 1 \\
\hline p30 & 6 & 2.5 & 6 & 6 & 10 & 9 & 15 & 1 \\
\hline p 40 & 7 & 3 & 7 & 8 & 14 & 12 & 15 & 1 \\
\hline p50 & 8 & 3 & 8 & 11 & 17 & 15 & 15 & 2 \\
\hline p60 & 9 & 4 & 13 & 13 & 23 & 19 & 15 & 3 \\
\hline p70 & 9 & 4.5 & 15.5 & 20 & 27 & 22 & 15 & 3 \\
\hline p80 & 10 & 7 & 16 & 21.2 & 30 & 24 & 15 & 4 \\
\hline p90 & 10 & 8 & 20 & 24 & 30 & 27 & 15 & 5 \\
\hline p100 & 10 & 10 & 21 & 30 & 30 & 30 & 15 & 5 \\
\hline \multicolumn{9}{|l|}{$5(n=112)$} \\
\hline p10 & 5 & 2 & 5 & 3 & 5 & 4 & 6 & 1 \\
\hline p20 & 7 & 3 & 8 & 6 & 9 & 7 & 13 & 2 \\
\hline p30 & 8 & 4 & 12 & 8 & 14 & 11 & 15 & 3 \\
\hline p 40 & 9 & 4 & 15 & 13 & 20 & 16 & 15 & 3 \\
\hline p50 & 10 & 4 & 17 & 17 & 24 & 21 & 15 & 4 \\
\hline p60 & 10 & 5 & 26 & 20 & 28 & 25 & 15 & 4 \\
\hline p70 & 10 & 6 & 30 & 30 & 30 & 28 & 15 & 4 \\
\hline p80 & 10 & 6 & 30 & 30 & 30 & 30 & 15 & 5 \\
\hline p90 & 10 & 7 & 30 & 30 & 30 & 30 & 15 & 5 \\
\hline p100 & 10 & 9 & 30 & 30 & 30 & 30 & 15 & 5 \\
\hline \multicolumn{9}{|l|}{$6(n=166)$} \\
\hline p10 & 6.7 & 2 & 3.7 & 5 & 7 & 4 & 10 & 1 \\
\hline p20 & 9 & 3 & 7 & 9 & 14 & 8 & 15 & 2 \\
\hline p30 & 9 & 4 & 9.3 & 13 & 19 & 13 & 15 & 2 \\
\hline $\mathrm{p} 40$ & 10 & 4 & 15 & 16.8 & 23 & 17 & 15 & 3 \\
\hline p50 & 10 & 5 & 20 & 20.1 & 27 & 21 & 15 & 4 \\
\hline p60 & 10 & 6 & 23.2 & 30 & 30 & 25 & 15 & 5 \\
\hline p70 & 10 & 7 & 30 & 30 & 30 & 29 & 15 & 5 \\
\hline p80 & 10 & 7 & 30 & 30 & 30 & 30 & 15 & 5 \\
\hline p90 & 10 & 8 & 30 & 30 & 30 & 30 & 15 & 5 \\
\hline p100 & 10 & 10 & 30 & 30 & 30 & 30 & 15 & 5 \\
\hline
\end{tabular}




\section{Discussion}

This is the first study to describe fitness levels and gross motor skills in children from 4 to 6 years old, from La Araucanía, Chile. In addition to providing percentile values by age and gender, the results will complement existing information for health parameters such as BMI, waist circumference, weight, and height [41].

The results of this study show that, regarding fitness level, boys obtained better results in tests of strength, while girls obtained better results in flexibility. These results were consistent with those reported in similar studies $[23,24]$, and are largely explained by the physical differences in muscle mass and height between genders [28]. For estimated VO2max, there were no significant differences by gender at 4 years, whereas at 5 years there were significant differences in favour of boys, and at 6 years in favour of girls. Other previous studies did not report these differences in favour of girls [27,35,42-44], which could be because the maximum values of $\mathrm{VO} 2 \mathrm{max}$ are relative $(\mathrm{mL} / \mathrm{kg} / \mathrm{min})$ and these differences could be explained by weight; however, at 6 years old the differences are minimal $(0.1 \mathrm{~kg})$, which could explain that the value in favour of girls is a consequence of increased physical activity that would benefit an increase in $\mathrm{VO} 2 \mathrm{max}$.

Regarding flexibility, there was an improvement from 4 to 5 years, but a decrease at 6 years, which would be in line with findings from a research carried out with children older than those evaluated in this study [13,27], where there was a progressive decline in this ability. However, this decrease may not only be explained by age, but also by scarce or no training carried out in this ability, since the training of flexibility in a specific manner will lead to its maintenance or improvement, promoting coordination and range of movements [13,19].

For gross motor skills, boys had better values in the catching and aiming tests, while girls obtained better results for static and dynamic balance, which is consistent with findings from other studies that used similar tests $[27,43,44]$.

In general, at a higher age, higher values for both genders were clear, and differences in favour of boys started to increase, which is in line with other studies $[22,24,27,28]$. These differences between genders, both for fitness level and gross motor skills, might not be explained by biological factors [8] alone, but also by socialization processes, where the type, duration, and frequency of the physical activities performed are different for the two genders [45], which is supported by Bandura [46], who states that school-age children are stereotyped in certain roles based on their gender. This is supported by noticing that it is common to see boys playing games or exercises with a ball while girls carry out activities and games with an expressive-rhythmic-motor component $[47,48]$. However, currently we should now consider whether these stereotypes are changing, and whether this may or may not be related to the significant differences in favour of girls at 6 years, which were found in relation to estimated $\mathrm{VO} 2 \mathrm{max}$.

Therefore, the results of this research will constitute an instrument that can be added to those that already exist for detecting and monitoring the health of children; providing an adequate basis for carrying out physical activity performance interventions, especially those aimed at the development of fitness levels and gross motor skills; and helping to prevent and treat diseases that currently affect the health of the population. In this sense, the schools play an important role since they are institutions where children spend a great deal of their time. Also, it has been demonstrated through meta-analyses [49] that various promotive strategies for physical activity in schools have been effective, and that these physical activity interventions should be promoted by educational institutions, combining with families and government agencies entrusted with public health. Thus, it is recommended that from an early age, girls and boys are encouraged to perform in different types of physical activity where there is no sex-based exclusion, which favours a similar development in physical condition and motor competence.

The potential limitations of this study are: (i) its transversal nature, in the absence of other variables that could give a greater understanding of the phenomenon and establish cause and effect relationships; (ii) the sample is not probabilistic and comes from only one region of Chile, so inferences 
to Chilean children should be made with caution. Therefore, more studies are needed at the national level that provide data to verify what is found here. However, the inclusion of poorly or barely studied ages could be a good first approach that may be useful for other research or health interventions.

It would be advisable to carry out further studies to deepen the research on this topic, incorporating variables such as diet, physical activity during free time, as well as the consideration of stereotypes (that change over time), and culture.

\section{Conclusions}

Our findings show that for fitness level and gross motor skills, boys have better values than girls in strength, catching and aiming tests, while girls have better results in flexibility and balance. Additionally, depending on age, the estimated VO2max shows significant differences in favour of boys or girls. No statistically significant differences in speed/agility are shown by gender. The study provides specific reference values for children aged 4 to 6 years by age and gender, which will aid physical education and health professionals in identifying those with low physical fitness and gross motor skills values, in order to establish goals to help improve their health, whereas for those with high values in abilities considered important for sports, this will help in early detection of higher competence.

Supplementary Materials: The following are available online at http:/www.mdpi.com/1660-4601/17/3/797/s1.

Author Contributions: All authors have contributed substantially to the work. In particular, (1) A.G.-C., P.F.-M., and J.B.-C. contributed to the conception and design of the study, data collection or analysis and interpretation of data; (2) A.G.-C., J.V.-G., I.C.-R., C.A.-B., and M.S.-L. contributed to the writing of the manuscript or revised it critically; and (3) A.G.-C., C.A.-B., and I.C.-R. approved the final version of the manuscript. All authors have read and agreed to the published version of the manuscript.

Funding: This research received no external funding.

Conflicts of Interest: The authors declare no conflicts of interest.

\section{References}

1. Ortega, F.B.; Ruiz, J.R.; Castillo, M.J.; Sjöström, M. Physical fitness in childhood and adolescence: A powerful marker of health. Int. J. Obes. 2008, 32, 1-11. [CrossRef] [PubMed]

2. Ruiz, J.R.; Castro-Piñero, J.; Artero, E.G.; Ortega, F.B.; Sjöström, M.; Suni, J.; Castillo, M.J. Predictive validity of health-related fitness in youth: A systematic review. Br. J. Sports Med. 2009, 43, 909-923. [CrossRef] [PubMed]

3. Janz, K.F.; Dawson, J.D.; Mahoney, L.T. Increases in physical fitness during childhood improve cardiovascular health during adolescence: The Muscatine Study. Int. J. Sport Med. Suppl. 2002, 23, 15-21. [CrossRef] [PubMed]

4. Castillo-Garzón, M.; Ruiz, J.R.; Ortega, F.B.; Gutiérrez-Sainz, Á. A Mediterranean Diet Is Not Enough for Health: Physical Fitness Is an Important Additional Contributor to Health for the Adults of Tomorrow. World Rev. Nutr. Diet. 2007, 97, 114-138. [PubMed]

5. Vicente-Rodriguez, G.; Ara, I.; Perez-Gomez, J.; Serrano-Sanchez, J.A.; Dorado, C.; Calbet, J.A.L. High femoral bone mineral density accretion in prepubertal soccer players. Med. Sci. Sports Exerc. 2004, 36, 1789-1795. [CrossRef] [PubMed]

6. Jankowski, M.; Niedzielska, A.; Brzezinski, M.; Drabik, J. Cardiorespiratory fitness in children: A simple screening test for population studies. Pediatr. Cardiol. 2015, 36, 27-32. [CrossRef]

7. Ruiz, J.R.; Cavero-Redondo, I.; Ortega, F.B.; Welk, G.J.; Andersen, L.B.; Martínez-Vizcaino, M. Cardiorespiratory fitness cut points to avoid cardiovascular disease risk in children and adolescents; what level of fitness should raise a red flag? A systematic review and meta-analysis. Br. J. Sports Med. 2017, 50, 1451-1458. [CrossRef]

8. Malina, R.; Bouchard, C.; Bar-Or, O. Growht, Maturation, and Physical Activity; Human Kinetics: Champaign, IL, USA, 2004.

9. Bai, Y.; Chen, S.; Laurson, K.R.; Kim, Y.; Saint-Maurice, P.F.; Welk, G. The Associations of Youth Physical Activity and Screen Time with Fatness and Fitness: The 2012 NHANES National Youth Fitness Survey. PLoS ONE 2016, 11, e0148038. [CrossRef] 
10. Valero, F.J.R.; Gualteros, J.A.; Torres, J.A.; Espinosa, L.M.U.; Ramírez-Vélez, R. Asociación entre el desempeño muscular y el bienestar físico en niños y adolescentes de Bogotá, Colombia. Nutr. Hosp. 2015, 32, 1559-1566.

11. Gomes, T.N.; dos Santos, F.K.; Katzmarzyk, P.T.; Maia, J. Active and strong: Physical activity, muscular strength, and metabolic risk in children. Am. J. Hum. Biol. 2017, 29, e22904. [CrossRef]

12. Díez-Fernández, A.; Martínez-Vizcaíno, V.; Torres-Costoso, A.; Cañete, J.; Franquelo-Morales, P.; Sánchez-López, M. Strength and cardiometabolic risk in young adults: The mediator role of aerobic fitness and waist circumference. Scand. J. Med. Sci. Sport 2018, 28, 1801-1807. [CrossRef] [PubMed]

13. Lopes, L.; Póvoas, S.; Mota, J.; Okely, A.D.; Coelho-e-Silva, M.J.; Cliff, D.P.; Lopes, V.P.; Santos, R. Flexibility is associated with motor competence in schoolchildren. Scand. J. Med. Sci. Sport 2017, 27, 1806-1813. [CrossRef] [PubMed]

14. Cadenas-Sanchez, C.; Intemann, T.; Labayen, I.; Peinado, A.B.; Vidal-Conti, J.; Sanchis-Moysi, J.; Moliner-Urdiales, D.; Rodríguez, M.A.; Cañete, J.; Fernández-Santos, J.D.R.; et al. Physical fitness reference standards for preschool children: The PREFIT project. J. Sci. Med. Sport 2019, 22, 430-437. [CrossRef] [PubMed]

15. Aubert, S.; Aguilar-Farías, N.; Cardon, G.; Chang, C.K.; Delisie, C.; Demetriou, Y.; Edwards, L.; Emeljanovas, A.; Gába, A.; Huang, W.Y.; et al. Report card grades on the physical activity of children and youth comparing 30 very high human development index countries. J. Phys. Act Health 2018, 15, 298-314. [CrossRef]

16. Aguilar-Farías, N.; Miranda-Marquez, S.; Sadarangani, K.P.; Martino Fuentealba, P.; Cristi-Montero, P.; Carcamo-Oyarzun, J.; Delgado-Floody, P.; Chandia-Poblete, D.; Rodríguez-rodríguez, F.; Mella-García, C.; et al. Results from Chile's 2018 Report Card on Physical Activity for Children and Youth. J. Phys. Act. Health 2018, 15, 298-331. [CrossRef]

17. D’Hondt, E.; Deforche, B.; Gentier, I.; De Bourdeaudhuij, I.; Vaeyens, R.; Philippaerts, R.; Lenoir, M. A longitudinal analysis of gross motor coordination in overweight and obese children versus normal-weight peers. Int. J. Obes. 2013, 37, 61-67. [CrossRef]

18. Piek, J.P.; Baynam, G.B.; Barrett, N.C. The relationship between fine and gross motor ability, self-perceptions and self-worth in children and adolescents. Hum. Mov. Sci. 2006, 25, 65-75. [CrossRef]

19. Lopes, L.; Santos, R.; Pereira, B.; Lopes, V.P. Associations between gross Motor Coordination and Academic Achievement in elementary school children. Hum. Mov. Sci. 2013, 32, 9-20. [CrossRef]

20. Stodden, D.F.; Langendorfer, S.J.; Goodway, J.D.; Langendorfer, S.J.; Roberton, M.; Rudisill, M.; García, C.; García, L. A developmental perspective on the role of motor skill competence in physical activity: An emergent relationship. Quest 2008, 60, 290-306. [CrossRef]

21. Loprinzi, P.; Cardinal, B.; Loprinzi, K.; Lee, H. Benefits and Environmental Determinants of Physical Activity in Children and Adolescents. Obes. Facts 2012, 5, 579-610. [CrossRef]

22. Amador-Ruiz, S.; Gutierrez, D.; Martínez-Vizcaíno, V.; Gulías-González, R.; Pardo-Guijarro, M.J.; Sánchez-López, M. Motor Competence Levels and Prevalence of Developmental Coordination Disorder in Spanish Children: The MOVI-KIDS Study. J. Sch. Health 2018, 88, 538-546. [CrossRef] [PubMed]

23. Vameghi, R.; Shams, A.; Dehkordi, P.S. The effect of age, sex and obesity on fundamental motor skills among 4 to 6 years-old children. Pakistan J. Med. Sci. 2012, 29, 586-590. [CrossRef] [PubMed]

24. Latorre, P.; Moreno, R.; Lucena, M.; Salas, J.; García-Pinillos, F.; Mora, D. Physical fitness in preschool children: Association with sex, age and weight status. Child Care Health Dev. 2017, 43, 267-273. [CrossRef] [PubMed]

25. Giagazoglou, P.; Kabitsis, N.; Kokaridas, D.; Zaragas, C.; Katartzi, E.; Kabitsis, C. The movement assessment battery in Greek preschoolers: The impact of age, gender, birth order, and physical activity on motor outcome. Res. Dev. Disabil. 2011, 32, 2577-2582. [CrossRef]

26. Cieśla, E. Physical fitness and motor skills in 6-year-old children from the Małopolska province. Kinesiology 2014, 24, 29-37. [CrossRef]

27. Gulías-González, R.; Sánchez-López, M.; Olivas-Bravo, Á.; Solera-Martínez, M.; Martínez-Vizcaíno, V. Physical fitness in spanish schoolchildren aged 6-12 years: Reference values of the battery EUROFIT and associated cardiovascular risk. J. Sch. Health 2014, 84, 625-635. [CrossRef]

28. Catley, M.J.; Tomkinson, G.R. Normative health-related fitness values for children: Analysis of 85347 test results on 9-17-year-old Australians since 1985. Br. J. Sports Med. 2013, 47, 98-108. [CrossRef] 
29. Vandorpe, B.; Vandendriessche, J.; Lefevre, J.; Pion, J.; Vaeyens, R.; Matthys, S.; Philippaerts, R.; Lenoir, M. The KörperkoordinationsTest für Kinder: Reference values and suitability for 6-12-year-old children in Flanders. Scand. J. Med. Sci. Sport 2011, 21, 378-388. [CrossRef]

30. Cadenas-Sánchez, C.; Alcántara-Moral, F.; Sánchez-Delgado, G.; Mora-González, J.; Martínez-Téllez, B.; Herrador-Colmenero, M.; Jiménez-Pavón, D.; Femia, P.; Ruiz, J.R.; Ortega, F.B. Assesment of cardiorespiratory fitness in preschool children: Adaptation of the 20 metres shuttle run test. Nutr. Hosp. 2014, 30, 1333-1343.

31. Mora-González, J.; Cadenas-Sánchez, C.; Martínez-Téllez, B.; Sánchez-Delgado, G.; Ruiz, J.R.; Léger, L.; Ortega, F.B. Estimating V02max in children aged 5-6 years through the preschool-adapted 20-m shuttle-run test (PREFIT). Eur. J. Appl. Physiol. 2017, 117, 2295-2307. [CrossRef]

32. Ortega, F.B.; Cadenas-Sánchez, C.; Sánchez-Delgado, G.; Mora-González, J.; Martínez-Telles, B.; Artero, E.G.; Castro-Piñero, J.; Labayen, I.; Chillón, P.; Löf, M.; et al. Systematic Review and Proposal of a Field-Based Physical Fitness-Test Battery in Preschool Children: The PREFIT Battery. Sport Med. 2015, 45, 533-555. [CrossRef] [PubMed]

33. Research CES. EUROFIT: Handbook for the EUROFIT Test of Physical Activity, 2nd ed.; Committee of Experts on Sports Research: Strasbourg, France, 1993.

34. Sanchez-Delgado, G.; Cadenas-Sanchez, C.; Mora-Gonzalez, J.; Martínez-Telles, B.; Chillón, P.; Löf, M.; Ruiz, J.R. Assessment of handgrip strength in preschool children aged 3 to 5 years. J. Hand Surg. Eur. Vol. 2015, 40, 966-972. [CrossRef] [PubMed]

35. Cadenas-Sanchez, C.; Martinez-Tellez, B.; Sanchez-Delgado, G.; Mora-Gonzalez, J.; Castro-Piñero, J.; Löf, M.; Ruiz, J.R.; Ortega, F.B. Assessing physical fitness in preschool children: Feasibility, reliability and practical recommendations for the PREFIT battery. J. Sci. Med. Sport 2016, 19, 910-915. [CrossRef] [PubMed]

36. Henderson, S.; Sudgen, D.; Barnett, A. Movement Assesment Battery for Children-2 Examiner's Manual; Harcourt Assesment: London, UK, 2007.

37. Niño-Cruz, G.; Camargo-Lemos, D.; Velásquez-Escobar, L.; Rodríguez-Ortiz, J.; Patiño-Segura, M. Age band 1 of the movement assessment battery for children-2. Reliability of the spanish version. Rev. Chil. Pediatr. 2019, 90, 522-532.

38. Ploegmakers, J.J.; Hepping, A.M.; Geertzen, J.H.; Bulstra, S.K.; Stevens, M. Grip strength is strongly associated with height, weight and gender in childhood: A cross sectional study of 2241 children and adolescents providing reference values. J. Physiotherp. 2013, 59, 255-261. [CrossRef]

39. Molenaar, H.M.; Selles, R.W.; Zuidam, J.M.; Willemsen, S.P.; Stam, H.J.; Hovius, S.E. Growth diagrams for grip strength in children. Clin. Orthop. Relat. Res. 2010, 468, 217-223. [CrossRef] [PubMed]

40. Wind, A.E.; Takken, T.; Helders, P.J.; Engelbert, R.H. Is grip strength a predictor for total muscle strength in healthy children, adolescents, and young adults? Eur. J. Peditr. 2010, 169, 281-287. [CrossRef] [PubMed]

41. Rodríguez, L.; Herrera, Y.; Leyton, C.; Pinheiro, A. Patrones De Crecimiento Para La Evaluación Nutricional de Niños, Niñas y Adolescentes, Desde El Nacimiento Hasta Los 19 Años de Edad; Ministerio de Salud: Santiago, Chile, 2018.

42. Redondo-Tébar, A.; Ruíz-Hermosa, A.; Martínez-Vizcaíno, V.; Cobo-Cuenca, A.; Bermejo-cantarero, A.; Cavero-Redondo, I.; Sánchez-López, M. Associations between health-related quality of life and physical fitness in 4-7-year-old Spanish children: The MOVIKIDS study. Qual. Life Res. 2019, 28, 1751-1759. [CrossRef]

43. Martínez-Vizcaíno, V.; Pozuelo-Carrascosa, D.P.; García-Prieto, J.C.; Cavero-Redondo, I.; Solera-Martínez, M.; Garrido-Miguel, M.; Diez-Fernández, A.; Ruiz-Hermosa, A.; Sánchez-López, M. Effectiveness of a school-based physical activity intervention on adiposity, fitness and blood pressure: MOVI-KIDS study. Br. J. Sports Med. 2018. [CrossRef]

44. Ruiz-Hermosa, A.; Martínez-Vizcaíno, V.; Alvarez-Bueno, C.; García-Prieto, J.C.; Pardo-Guijarro, M.J.; Sánchez-López, M. No Association between Active Commuting to School, Adiposity, Fitness, and Cognition in Spanish Children: The MOVI-KIDS Study. J. Sch. Health 2018, 88, 839-846. [CrossRef]

45. Hardy, L.L.; Reinten-Reynolds, T.; Espinel, P.; Zask, A.; Okely, A.D. Prevalence and correlates of low fundamental movement skill competency in children. Pediatrics 2012, 130, 390-398. [CrossRef] [PubMed]

46. Bandura, A. Social Foundations of Thought and Action: A Social Cognitive Perspective; Prentice-Hall: Englewood Cliffs, NJ, USA, 1986. 
47. Resaland, G.K.; Aadland, E.; Andersen, J.R.; Bartholomew, J.B.; Anderssen, S.A.; Moe, V.F. Physical activity preferences of 10-year-old children and identified activities with positive and negative associations to cardiorespiratory fitness. Acta Paediatr. 2019, 108, 354-360. [CrossRef] [PubMed]

48. Martínez-Andrés, M.; García-López, U.; Gutiérrez-Zornoza, M.; Rodríguez-Martín, B.; Pardo-Guijarro, M.J.; Sánchez-López, M.; Cortez-Ramírez, E.; Martínez-Vizcaíno, V. Barries, facilitators and preferences for the physical activity of school children. Rationale and methods of a mixed study. BMC Public Health 2012, 12, 785. [CrossRef] [PubMed]

49. Pozuelo-Carrascosa, D.P.; Cavero-Redondo, I.; Herraiz-Adillo, A.; Diez-Fernandez, A.; Sánchez-López, M.; Martinez-Vizcaino, V. School-based exercise programs and cardiometabolic risk factors: A meta-analysis. Pediatrics 2018, 142, e20181033. [CrossRef]

(C) 2020 by the authors. Licensee MDPI, Basel, Switzerland. This article is an open access article distributed under the terms and conditions of the Creative Commons Attribution (CC BY) license (http://creativecommons.org/licenses/by/4.0/). 